\title{
Chirality distribution and transition energies of carbon nanotubes
}

\author{
H. Telg, ${ }^{1}$ J. Maultzsch, ${ }^{1}$ S. Reich, ${ }^{2}$ F. Hennrich, ${ }^{3}$ and C. Thomsen ${ }^{1}$ \\ ${ }^{1}$ Institut für Festkörperphysik, Technische Universität Berlin, Hardenbergstr. 36, 10623 Berlin, Germany \\ ${ }^{2}$ Department of Engineering, University of Cambridge, Cambridge CB2 1PZ, United Kingdom \\ ${ }^{3}$ Institut für Nanotechnologie, Forschungszentrum Karlsruhe, 76021 Karlsruhe, Germany
}

(Dated: November 11, 2018)

\begin{abstract}
From resonant Raman scattering on isolated nanotubes we obtained the optical transition energies, the radial breathing mode frequency and Raman intensity of both metallic and semiconducting tubes. We unambiguously assigned the chiral index $\left(n_{1}, n_{2}\right)$ of $\approx 50$ nanotubes based solely on a third-neighbor tight-binding Kataura plot and find $\omega_{\mathrm{RBM}}=(214.4 \pm 2) \mathrm{cm}^{-1} \mathrm{~nm} / d+(18.7 \pm 2) \mathrm{cm}^{-1}$. In contrast to luminescence experiments we observe all chiralities including zig-zag tubes. The Raman intensities have a systematic chiral-angle dependence confirming recent ab-initio calculations.

PACS numbers: 78.67.Ch, 73.22.-f, 78.30.Na
\end{abstract}

The successful preparation of single-walled carbon nanotubes in solution where the tubes are prevented from rebundling has opened a new direction in carbon nanotube research [1, 2, 3, 4]. Strong luminescence by direct recombination from the band gap was detected in these isolated tubes, whereas in nanotube bundles no luminescence is observed. The electronic structure of carbon nanotubes and the optical transition energies vary strongly with their chiral index $\left(n_{1}, n_{2}\right)[\underline{5}]$. Because the synthesis of nanotubes with a predefined chiral index has not been achieved so far, luminescence experiments were carried out on tube ensembles with unknown composition of chiral angles. Several attempts to assign the chiral index $\left(n_{1}, n_{2}\right)$ to the experimentally observed luminescence peaks were reported [2, 4, 6, 7]. With a unique assignment, one could validate and possibly revise theoretical models of the electronic band structure. Moreover, such an assignment would allow to characterize the tubes after their production and to control their separation [8].

Bachilo et al. suggested an $\left(n_{1}, n_{2}\right)$ assignment of the first and second transition energies in semiconducting tubes 2]. Their assignment is based on pattern recognition between experiment and theory in a plot of the second transition (excitation energy) versus the first transition (emission energy) 9]. The patterns, however, were not unique, and the frequency of the radial breathing mode (RBM) was used to find an anchoring element that singles out one of the assignments. Surprisingly, zig-zag tubes were not detected in these luminescence experiments. Bachilo et al. concluded that the concentration of tubes with chiral angles close to the zig-zag direction was very low in the sample [2].

The electronic transition energies of metallic nanotubes cannot be detected by luminescence experiments. An elegant approach is to record Raman resonance profiles 10, 11, 12, 13], with maximum intensity close to the real transitions in the electronic band structure. Resonance profiles from nanotubes in solution were first reported by Strano et al. [14]; their $\left(n_{1}, n_{2}\right)$ assignment to the transition energies was based on the RBM frequency to tube diameter relationship of Ref. [2]. The resonance profiles of the so-assigned RBM peaks were then used to find an empirical expression for the transition energies in metallic tubes.

In this paper we present the transition energies of both metallic and semiconducting nanotubes by resonant Raman spectroscopy. Plotting the resonance maxima as a function of inverse RBM frequency, we obtain an $\left(n_{1}, n_{2}\right)$ assignment without any additional assumptions. From our assignment we fit $c_{1}=214.4 \mathrm{~cm}^{-1} \mathrm{~nm}$ and $c_{2}=18.7 \mathrm{~cm}^{-1}$ for the relation between diameter and RBM frequency. We observed several semiconducting tubes that were not detectable by luminescence. Our results show that the electron-phonon coupling strength increases systematically for smaller chiral angles. Conclusions about the distribution of chiral angles in a sample based solely on luminescence intensity lead to incorrect results; in particular, zig-zag tubes are present in nanotube ensembles.

We performed Raman spectroscopy on HiPCO nanotubes with diameters $d \approx 0.7-1.2 \mathrm{~nm}[15]$. We dispersed the tubes in $\mathrm{D}_{2} \mathrm{O}$ containing a surfactant, see Ref. [4] and used an Ar-Kr laser between 2.18 and $2.62 \mathrm{eV}$ and two tunable lasers $(1.85-2.15 \mathrm{eV}$ and $1.51-1.75 \mathrm{eV})$. The spectra were collected with a Dilor XY800 spectrometer in backscattering geometry at room temperature. To obtain the Raman cross section from the measured intensity we normalized the spectra to $\mathrm{CaF}_{2}$ and $\mathrm{BaF}_{2}$ measurements taken under the same experimental conditions (integration time, laser power). This also corrects for the spectrometer sensitivity and the $\omega^{4}$ dependence of the Raman process. The Raman susceptibility was calculated from the normalized spectra by dividing by the Bose-Einstein occupation number and the inverse phonon frequency [16]. The latter was omitted in Fig. 团(a) for a better representation.

In Fig. 1(a) we show a contour plot of all Raman spectra, i.e., the Raman scattering power as a function of inverse RBM frequency $\left(1 / \omega_{\mathrm{RBM}}\right)$ and excitation energy. When tuning the excitation energy, the RBM peaks 

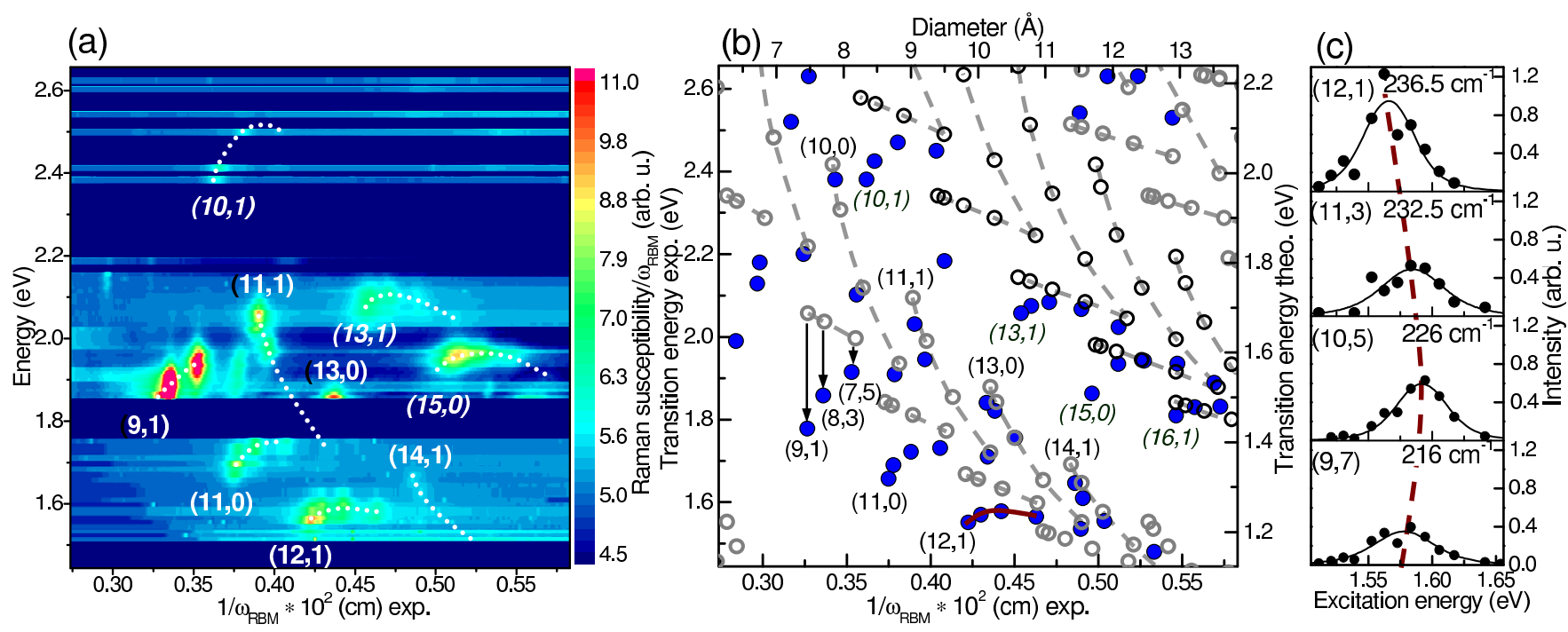

FIG. 1: (Color online) (a) Contour plot of the Raman cross section of the RBM as a function of excitation energy and reciprocal RBM frequency. In (a) and (b) the dotted and dashed lines connect maxima originating from tubes of the same branch. In each branch the member with the largest RBM frequency is labeled. (b) Kataura plot from experimental results (filled circles) and third-neighbor tight-binding calculations (open circles). Gray and black indicate semiconducting (2nd and 3rd transitions) and metallic tubes, respectively. The plot consists of theoretical (right, top axes) and experimental data (left, bottom axes). The error in experimental transition energies is mostly smaller than $0.02 \mathrm{eV}$. For the $(9,1)$ branch, the vertical arrows indicate for each member the assignment to its theoretically predicted point. The increasing softening compared to the theoretical transition energies with smaller chiral angle is clearly seen. (c) Integrated Raman intensity as a function of excitation energy for the tubes in the $(12,1)$ branch.

appear and disappear in groups of close-by frequencies. These are indicated by the dotted lines, where the laterassigned chiral index of the largest RBM frequency of each group is given. To obtain the optical transition energies, we use the resonance profiles, see, e.g., Fig. 团(c) for the group beginning with the $(12,1)$ tube. The lines are fits to the first-order Raman cross section including incoming and outgoing resonance. From these fits we obtain directly the transition energies, which are by $\approx 10 \mathrm{meV}\left(0.5 \omega_{\mathrm{RBM}}\right)$ smaller than the energy of the resonance maximum. For decreasing RBM frequency, the resonance energy first increases and then decreases again [dashed line in Fig. 1(c)]. The maximum intensity decreases with decreasing RBM frequency. The described systematics are valid only for RBMs within a given group of tubes defined by the dotted lines in Fig. 1(a).

In Fig. 1(b) we plot the experimental transition energies as a function of $1 / \omega_{\text {RBM }}$ (solid circles, bottom and left axes). Since $1 / \omega_{\mathrm{RBM}}$ is approximately proportional to the tube diameter, we thereby obtain an experimental Kataura plot, which we compare to thirdnearest neighbor tight-binding calculations [9] (open circles, upper and right axes). For semiconducting tubes, the measured transition energies are in excellent agreement with the $E_{22}$ energies measured by Bachilo et al. 2]. From a comparison between the experimental and the calculated Kataura plot we were able to assign the tube chiralities in the following way. Since we did not want to make any assumptions about the coefficients $c_{1}$ and $c_{2}$ in the diameter-RBM frequency relationship, $\omega_{\mathrm{RBM}}=\left(c_{1} / d+c_{2}\right)$, we varied $c_{1}$ and $c_{2}$ to find the best match between experimental and calculated data in Fig. 1(b). Varying $c_{1}$ and $c_{2}$, respectively, corresponds to stretching and displacing the theoretical Kataura plot along the $1 / \omega_{\text {RBM }}$ axis. In addition we displaced the plot on the energy scale. The origin of this overall energy offset is, among others, found in the tight-binding parameters, which were fitted to LDA calculations believed to underestimate the transition energies by typically $10 \%$.

Figure 1(b) shows the best $\left(n_{1}, n_{2}\right)$ assignment of experimental transition energies and RBM frequencies. The dashed lines in the theoretical Kataura plot indicate branches that are formed by tubes with $2 n_{1}+n_{2}$ constant. The indices of neighboring tubes in a given branch are related through $\left(n_{1}+1, n_{2}-2\right)$ or $\left(n_{1}-1, n_{2}+2\right)$, as long as $n_{1} \geq n_{2}$. For example, the branch starting with the $(11,0)$ tube contains from small to large diameter $(11,0),(10,2),(9,4)$, and $(8,6)$, and belongs to the $\nu=\left(n_{1}-n_{2}\right) \bmod 3=-1$ family. The chiral angle $\theta$ of the tubes within a branch increases towards larger diameter. Zig-zag tubes $\left(\theta=0^{\circ}\right)$ are always at the outermost position of a branch. The RBM groups in Fig. 1](a) [dotted lines] directly correspond to these branches, see, e.g., the one beginning with the $(9,1)$ tube.

We find a very good match between the patterns of the calculated diameter and of the experimental values 
TABLE I: Measured $\omega_{\mathrm{RBM}}$ and $E_{22}$ for the branch of the $(11,0)$ tube. See also supplementary material [17].

\begin{tabular}{lcccc}
\hline \hline chiral index & $(11,0)$ & $(10,2)$ & $(9,4)$ & $(8,6)$ \\
\hline$\omega_{\mathrm{RBM}}\left(\mathrm{cm}^{-1}\right)$ & 266.7 & 264.6 & 257.5 & 246.4 \\
$E_{22}(\mathrm{eV})$ & 1.657 & 1.690 & 1.72 & 1.73 \\
\hline \hline
\end{tabular}

$1 / \omega_{\text {RBM }}$. The experimental transition energies, however, deviate systematically from the calculations for the branches on the low-energy side of the semiconducting and of the metallic transitions. This deviation increases for smaller chiral angles. In other words, the experimental transition energies bend downwards from the calculated transitions with decreasing chiral angle. Such a strong deviation between theory and experiment is not seen for the upper Kataura branches, where the tubes belong to the $\nu=+1$ family. This was observed in luminescence experiments as well and will be discussed below.

We considered alternative assignments by displacing the experimental data along the sets of semiconducting and metallic tubes in Fig. 团(b) to the left and to the right. None of them yields a good agreement regarding the data points within the $\left(n_{1}-1, n_{2}+2\right)$ branches: Either the number of tubes differs between the theoretical and experimental branch or some points are eminently displaced horizontally from the theoretical ones. Having found the zig-zag tubes we can exclude these alternative assignments. Table凹summarizes the assignment of chiral indices to the measured RBM frequencies and transition energies for the branch of the $(11,0)$ tube [17].

From Fig. 1(b) we can now fit the coefficients of the linear relationship between $\omega_{\mathrm{RBM}}$ and $1 / d$ of the assigned nanotubes. The linear fit based on 45 identified tubes yields $\omega_{\mathrm{RBM}}=c_{1} / d+c_{2}$ with $c_{1}=(214.4 \pm 2) \mathrm{cm}^{-1} \mathrm{~nm}$ and $c_{2}=(18.7 \pm 2) \mathrm{cm}^{-1}$. Here, the tube diameter is geometrically determined by $d=a_{0} \sqrt{n_{1}^{2}+n_{1} n_{2}+n_{2}^{2}} / \pi$, using a graphite lattice constant $a_{0}=2.461 \AA$. $c_{1}$ and $c_{2}$ differ somewhat from the coefficients found in Ref. [2] for the same type of samples, because we used many more RBM frequencies for the fit and calculated the diameter from a smaller $a_{0}$. The coefficients are similar to theoretical predictions 18, 19 but different from other experimental work [12, 20]. In Ref. [12], $c_{1}$ and $c_{2}$ were used as free parameters to find the assignment; moreover, the experimental information on the transition energies was not included. In Ref. [20], the chiral index assignment also depends on $c_{1}\left(c_{2}=0\right)$. Moreover, the tubes are on a substrate, which might alter the RBM frequencies. In contrast, our assignment is not based on a choice for $c_{1}$ and $c_{2}$. For the first time, they are obtained by a linear fit after the assignment was performed. For example, the $(11,0)$ tube has an RBM frequency of $266.7 \mathrm{~cm}^{-1}$ and $E_{22}=1.657 \mathrm{eV}$ regardless of its exact diameter or any fitting procedure, see Table【
In addition to the semiconducting tubes, we directly obtained the transition energies of metallic tubes. Our assignment of the metallic tubes to RBM frequencies agrees well with Strano et al. 14. The empirically obtained transition energies in Ref. 14], however, underestimate the experimental values in Fig. 1(b). Moreover, our data show a stronger bending of the branches towards small chiral angles. This discrepancy comes mainly from the presence of pairs of close-by transition energies in chiral metallic tubes 21]. This ambiguity led to an incorrect assignment of single data points to the upper or lower transition.

Given the unique $\left(n_{1}, n_{2}\right)$ assignment of the RBM and the resonance energies, we can now examine the chirality dependence of the transition energies and of the RBM intensity. The optical transition energies of carbon nanotubes are roughly proportional to $1 / d$ 9, 21, 22, 23]. Tight-binding calculations predict deviations from a pure $1 / d$ dependence as a function of chiral angle [21]. They lead to the branches in the Kataura plot and are more pronounced if third-nearest neighbors are included [9]. Still, the band gap energies of tubes with small chiral angle (zig-zag tubes) are systematically lower in firstprinciples calculations than in zone folding [24]. The branches in the experimental Kataura plot of Fig. 1(b) are an experimental verification of the predictions from $a b$-initio calculations.

The chiral-angle dependent softening of the transition energies with respect to the third-order tight-binding calculations is due to rehybridization of the $\pi$ and $\sigma$ bands $9,24,25$. It is stronger for the states originating from between the $K$ and $M$ point of the graphite Brillouin zone in the zone-folding approach and weaker for states from the other side of the $K$ point 24]. In semiconducting tubes, the value of $\nu=\left(n_{1}-n_{2}\right) \bmod 3$ determines from which side of the $K$ point the electronic states originate for a given optical transition. The tubes in the lower branches of the $E_{22}$ transitions in semiconducting tubes, i.e., of the branches beginning with $(9,1)$, $(11,0)$, and $(12,1)$ in Fig. 团(b), have $\nu=-1$. The softening for small-chiral-angle tubes in these branches compared to the tight-binding value is quite strong. The tubes in the upper part of the same set of transitions have $\nu=+1$ and are less affected by the softening. For example, the experimental transition energy of the $(10,0)$ tube $\left[\theta=0^{\circ}, \nu=+1\right]$ matches the theoretical value very well [Fig. 1(b)]. Taking this softening of the transition energies in the -1 families into account, the agreement of our data with the theoretical predictions is excellent.

In general, the RBM signal was strong for nanotubes with $\nu=-1$ and also from the lower branches of the metallic transitions in Fig. 1(b). The intensities were by a factor of four to ten weaker for tubes with $\nu=+1$. This observation confirms ab-initio calculations of the electron-phonon coupling that predicted the magnitude of the electron-phonon matrix element to alternate with 


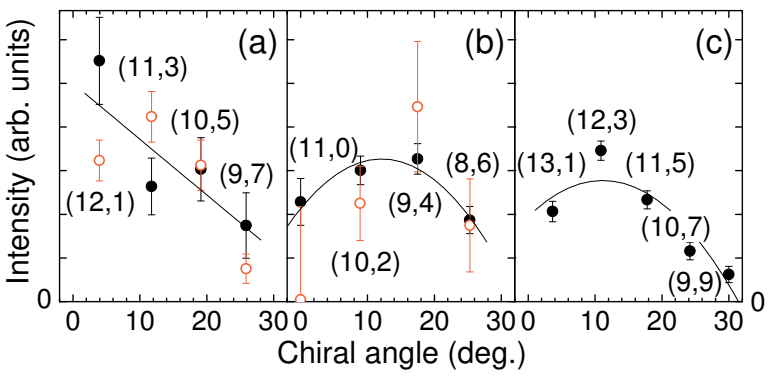

FIG. 2: (Color online) Raman intensity as a function of the chiral angle for three nanotube branches (solid circles) with $\left(n_{1}, n_{2}\right)$ as indicated. Open circles: calculated Raman intensity, see text. (a) and (b) contain semiconducting, (c) contains metallic tubes.

$\nu$ [26. The relative Raman intensity of the RBM can also be used to discriminate between the two families of semiconducting tubes.

The assignment of the semiconducting tubes in Fig. 1(b) corresponds to the one found by Bachilo et al. from luminescence experiments [2]. In contrast to the luminescence results, which reported a maximum intensity for close-to-armchair tubes and no emission from zig-zag tubes, we clearly observed zig-zag or close-to-zigzag tubes as well. The $(13,0),(11,0)$, and the $(10,0)$ tube show that zig-zag tubes are present in the sample. These tubes as well as the $(14,1)$ tube were not observed by photoluminescence. An important conclusion from our work is that the absence of photoluminescence from these tubes thus does not imply the preferential growth of armchair tubes.

In Fig. 2 we show the resonance maxima for tubes belonging to the same $\left(n_{1}-1, n_{2}+2\right)$ branch. The Raman intensity increases with decreasing chiral angle $\theta$ and is at maximum for $\theta \approx 10-15^{\circ}$, except for (a). Theoretically, the Raman amplitude is proportional to the electronphonon coupling times the square of the optical absorption strength [16]. Machón et al. 26] found that the electron-phonon coupling of the RBM decreases strongly with increasing $\theta$. We therefore model the electronphonon interaction by a linear function of the chiral angle with a three times stronger coupling for zig-zag than for armchair tubes as found in Ref. 26]. We approximate the optical absorption strength of a tube by its experimental photoluminescence intensity [2], i.e., we assume the absorption and

emission probability to be the same. The relative Raman intensities calculated with this model are in good agreement with experiment (open dots in Fig. 2). The Raman response of zig-zag tubes is enhanced compared to their luminescence intensity because of their strong electron-phonon coupling. On the other hand, the Raman intensity of zig-zag tubes is smaller than for $\theta \approx 10^{\circ}$ due to the small absorption coefficient. Thus, our data are completely consistent with a uniform chirality distri- bution in the sample.

In conclusion, we assigned the chiral indices to $\approx 50$ measured RBM frequencies and transition energies by resonant Raman spectroscopy. In contrast to all previous work our assignment is independent of the coefficients $c_{1}$ and $c_{2}$, which we fit only after assigning the chiral index to a particular RBM. The largest Raman intensity was measured for tubes with chiral angles around $15^{\circ}$ or smaller, which is in agreement with theoretical predictions and implies that the chiralities are evenly distributed. Moreover, our results confirm that the RBM intensity in semiconducting tubes depends on the $\left(n_{1}-n_{2}\right)$ mod 3 family. The transition energies deviate from zone-folding predictions with decreasing chiral angle, which, in particular for metallic tubes, was strongly underestimated in earlier work.

This work was supported by the DFG under grant number Th662/8-2. S. R. was supported by the Oppenheimer Fund and Newnham College.

[1] M. J. O'Connell, et al., Science 297, 593 (2002).

[2] S. M. Bachilo, et al., Science 298, 2361 (2002).

[3] J. Lefebvre, et al., Phys. Rev. Lett. 90, 217401 (2003).

[4] S. Lebedkin, et al., J. Phys. Chem. B 107, 1949 (2003).

[5] S. Reich, C. Thomsen, and J. Maultzsch, Carbon Nanotubes: Basic Concepts and Physical Properties (WileyVCH, Berlin, 2004).

[6] A. Hagen and T. Hertel, Nano Lett. 3, 383 (2003).

[7] R. B. Weisman and S. M. Bachilo, Nano Lett. 3, 1235 (2003).

[8] R. Krupke, F. Hennrich, H. v. Löhneysen, and M. M. Kappes, Science 301, 344 (2003).

[9] S. Reich, J. Maultzsch, C. Thomsen, and P. Ordejón, Phys. Rev. B 66, 035412 (2002).

[10] A. Jorio, et al., Phys. Rev. B 63, 245416 (2001).

11] M. Canonico, et al., Phys. Rev. B 65, 201402(R) (2002).

[12] C. Kramberger, et al., Phys. Rev. B 68, 235404 (2003).

[13] S. K. Doorn, et al., Appl. Phys. A 78, 1147 (2004).

[14] M. S. Strano, et al., Nano Lett. 3, 1091 (2003).

[15] P. Nikolaev, et al., Chem. Phys. Lett. 313, 91 (1999).

[16] M. Cardona, in Light Scattering in Solids II, edited by M. Cardona and G. Güntherodt (Springer, Berlin, 1982), vol. 50 of Topics in Applied Physics, p. 19.

[17] See EPAPS Document No. [] for a table containing the complete branches of Fig. 10(b).

[18] J. Kürti, G. Kresse, and H. Kuzmany, Phys. Rev. B 58, 8869 (1998).

[19] E. Dobardžić, I. M. B. Nikolić, T. Vuković, and M. Damnjanović, Phys. Rev. B 68, 045408 (2003).

[20] A. Jorio, et al., Phys. Rev. Lett. 86, 1118 (2001).

[21] S. Reich and C. Thomsen, Phys. Rev. B 62, 4273 (2000).

[22] J. W. Mintmire and C. T. White, Phys. Rev. Lett. 81, 2506 (1998).

[23] H. Kataura, et al., Synth. Met. 103, 2555 (1999).

[24] S. Reich, C. Thomsen, and P. Ordejón, Phys. Rev. B 65, 155411 (2002).

[25] X. Blase, et al., Phys. Rev. Lett. 72, 1878 (1994).

[26] M. Machón, et al., (2003), submitted to Phys. Rev. B; 
cond-mat/0408436 1. 